\title{
GENERATION OF THz RADIATION IN NONLINEAR CRYSTAL PARTIALLY FILLING A HOLLOW WAVEGUIDE
}

\author{
A.S. NIKOGHOSYAN \\ Department of Radiophysics, Yerevan State University, \\ Alex Manoogian 1,Yerevan, 0025 Armenia \\ nika@ysu.am
}

\begin{abstract}
The results of the THz pulse generation via optical rectification of pico- and femtosecond laser pulses in nonlinear crystal are presented. Detection of $\mathrm{THz}$ pulses is performed at room temperature by both Schottky diode and ZnTe crystal. The quantum efficiency of energy conversion up to $28 \%$ of maximum value is obtained with Schottky diode.
\end{abstract}

Keywords: Optical rectification; efficient $\mathrm{THz}$ pulse generation; rectangular waveguide; femtosecond laser, Schottky diode.

\section{Introduction}

In recent years considerable achievements were reached in development of sources and detectors in terahertz $(\mathrm{THz})$ band $(0.1-100 \mathrm{THz})$. They found various potential applications in terahertz time-domain spectroscopy (THz-TDS), medical diagnostics, remote sensing and security screening, space science, high-speed communications and high resolution of $\mathrm{THz}$ waveguide spectroscopy.

For transmitting the $\mathrm{THz}$ radiation from the source to the object and then to the detector it is necessary to utilize different guided structures, such as hollow metallic waveguides $^{1-3}$, solid wires ${ }^{4}$, copper coated flexible hollow polycarbonate waveguide ${ }^{5}$, metallic sl-3ot waveguide $^{6}$, one-dimensional (1D) Bragg waveguide ${ }^{7}$, parallel-plate waveguides $^{8}$, dielectric waveguide ${ }^{9}$, surface plasmon polariton waveguides ${ }^{10-11}$ and ribbon-based dielectric waveguides ${ }^{12}$.

The value of the generated terahertz $(\mathrm{THz})$ power limits the major specifications of the $\mathrm{THz}$ coherent technique applications in imaging and tomography, such as penetration depth, signal-to-noise ratio, and so on. Therefore the increase of generated $\mathrm{THz}$ power at room temperature will improve the overall performance of $\mathrm{THz}$ imaging systems.

During propagation of optical laser pulse in the medium with the second order nonlinear susceptibility a conversion of energy into radiation of sum and difference frequency owing to coherent mixing of spectral components of the optical pulse occurs. To effectively generate radiation sum or difference frequency, it is necessary to provide 
the phase-matching condition for all spectral components of the optical pulse participating in a nonlinear process. Phase matching between group velocity of the optical pulse and phase velocity of difference frequency wave is performed by different methods: birefringence, dispersion in the pump frequency range, wave front tilting, artificial periodic structure. The possibility of increasing light conversion efficiency into $\mathrm{THz}$ range by nonlinear crystal partially filling the metallic rectangular waveguide was suggested, theoretically grounded and experimentally realized in Ref. 2, 3, 13. The metallic rectangular hollow waveguide is used to achieve phase matching, large coupling coefficients, and low propagation loss for both the optical and $\mathrm{THz}$ waves. This method broadens the choice of nonlinear materials. Another advantage of this method is that by choosing the crystal thickness and its disposition concerning the narrow wall of the waveguide it is possible not only to control mode type, but also to make the absorption loss of the waveguide with the crystal much less than the intrinsic loss of the crystal.

\section{The Generation of THz Radiation in Nonlinear Crystal $\mathrm{LiNbO}_{3}$ Partially Filling the Metallic Rectangular Waveguide}

The optical excitation of the $\mathrm{LiNbO}_{3}$ crystal having tapered end is performed by 50 or $200 \mathrm{fs}$ pulses of a Ti:sapphire $\operatorname{laser}^{13}(\lambda=800 \mathrm{~nm})$ with frequency repetition of $82 \mathrm{MHz}$ or 1 ps pulses of a mode-locked Nd: glass $\operatorname{laser}^{2,3}(\lambda=1.06 \mu \mathrm{m})$ with pulse repetition period 7 ns. Time dependence of the $\mathrm{THz}$ field when $\mathrm{LiNbO}_{3}$ is placed in a free space (FS) and then in a hollow metallic rectangular waveguide (WG) $1.8 \times 0.9 \mathrm{~mm}^{2}$ is shown in Fig.1(a) and its corresponding spectra after Fast Fourier Transform (FFT) in Fig.1(b). Coherent detection $^{14}$ of linear polarized femtosecond $\mathrm{THz}$ pulse in the band $0.1-2.7 \mathrm{THz}$ as the superposition of $\mathrm{H}_{\text {no }}$ modes is performed with a dynamic electro-optical sampling cell (EOSC) consisting of the crystal ZnTe orientation (110) with $1 \mathrm{~mm}$ thickness.

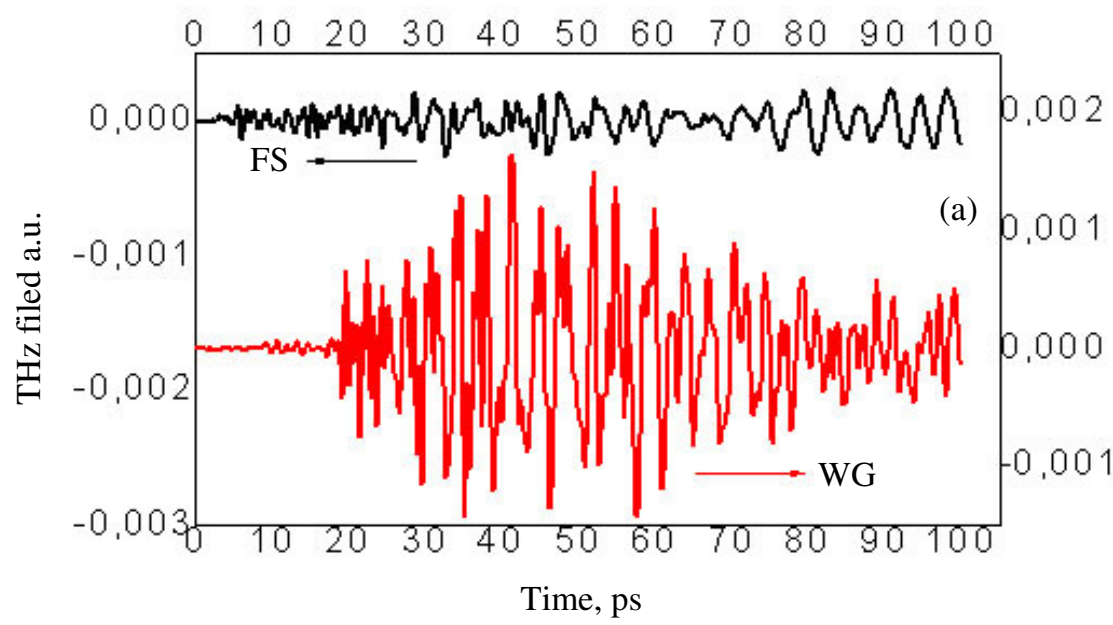




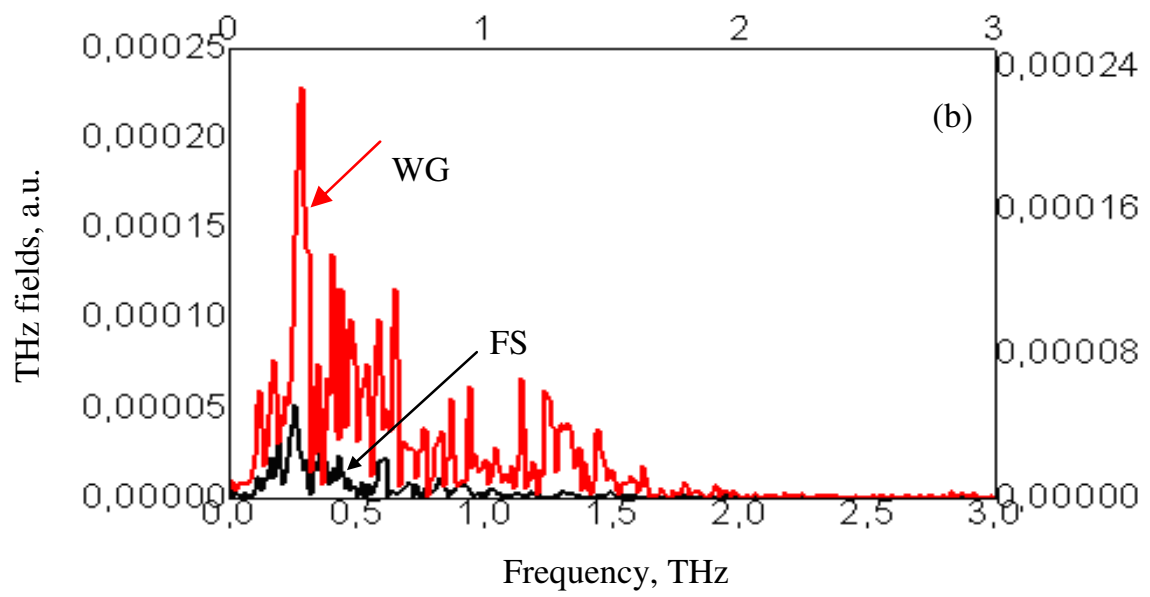

Fig.1. Time dependence (a) of the THz field and its spectrum (b) after fast Fourier transform when the crystal is placed in free space (FS) and centrally-symmetrically in the waveguide (WG) with dimensions $1.8 \times 0.9 \mathrm{~mm}^{2}$.

Nanostructured GaAs Schottky barrier diodes have been widely used not only as low noise mixers in high resolution heterodyne spectrometers in the spectral range $300 \mathrm{GHz}$ to $5 \mathrm{THz}(1-20 \mathrm{meV})$ and a spectral resolution of $v / \Delta v>10^{6}$, as well as frequency multipliers and detectors. Schottky diodes (rectifying metal-semiconductor junctions) are used as a detector because of their higher switching speeds and their inherent suitability for low-voltage. The high-frequency performance of GaAs Schottky barrier diodes is limited by the parasitic circuit elements within the diode, particularly the series impedance and junction capacitance. The cutoff frequency of GaAs diode ${ }^{15}$, specifically model $1 \mathrm{~T} 15$ is $25 \mathrm{THz}$ (Table 1).

Table 1. Schottky diode parameters.

\begin{tabular}{|c|c|c|c|c|c|c|c|}
\hline Diode & $\begin{array}{c}\text { Anode } \\
\text { Diamet. } \\
\mathrm{d}(\mu \mathrm{m})\end{array}$ & $\begin{array}{c}\text { Epilayer } \\
\text { Thickn. } \\
\mathrm{t}(\AA)\end{array}$ & $\begin{array}{c}\text { Series } \\
\text { Resist. } \\
\mathrm{R}(\Omega)\end{array}$ & $\begin{array}{c}\text { Zero-Bias } \\
\text { Capacit. } \\
\mathrm{C}(\mathrm{fF})\end{array}$ & $\begin{array}{c}\Delta \mathrm{V} \text { at } \\
10-100 \mu \mathrm{A} \\
(\mathrm{mV})\end{array}$ & $\begin{array}{c}\text { Ideality } \\
\text { Factor, } \eta \\
10-100 \mu \mathrm{A} \\
(\mathrm{mV})\end{array}$ & $\begin{array}{c}\text { Cut-off } \\
\text { Frequency } \\
v=1 / 2 \pi \mathrm{RC}, \\
(\mathrm{THz})\end{array}$ \\
\hline $1 \mathrm{~T} 14$ & 0.45 & 400 & $8-10$ & $0.9-1.1$ & $85-90$ & $1.4-15$ & 18 \\
\hline $1 \mathrm{~T} 15$ & 0.25 & 300 & 25 & 0.3 & 90 & 1.5 & 25 \\
\hline $1 \mathrm{~T} 6$ & 0.45 & 1000 & 40 & 0.4 & $81-83$ & $1.35-1.4$ & 10 \\
\hline $1 \mathrm{I} 7$ & 0.80 & 800 & $10-13$ & $0.8-1.4$ & 77 & 1.3 & 12.6 \\
\hline
\end{tabular}

The optimum current dependence on frequency for four diodes ${ }^{16}$ is shown in Fig.2. The local oscillator frequencies are used in the range of from $11 \mathrm{GHz}$ to $2550 \mathrm{GHz}$. 


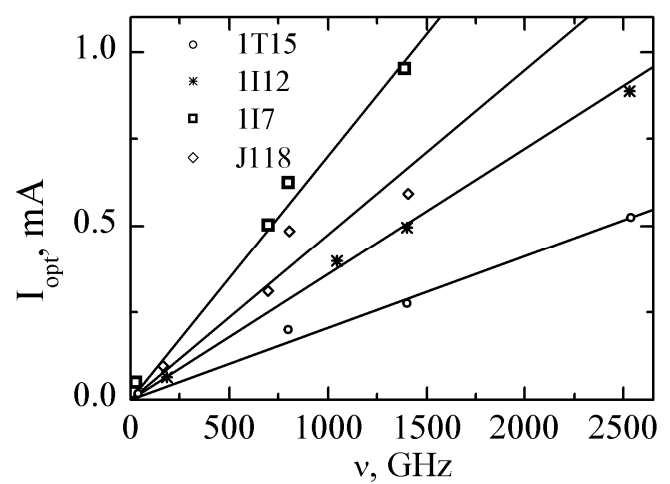

Fig.2. The optimum current dependence on frequency for diodes, showing the linear relationship for each of the diodes.

In our experiment the Schottky diode was used as a direct detector in broad frequency range with the cutoff frequency $\mathrm{f}_{\mathrm{c}}(\mathrm{GaAs}) \sim 7 \mathrm{THz}$ and with extremely fast response time $<1$ ps. Therefore the $\mathrm{THz}$ pulse train envelope reproduces the envelope of laser pulse train in the required frequency range at room temperature. The lengths of the utilized $\mathrm{LiNbO}_{3}$ crystal were changed from $0.4 \mathrm{~mm}$ to $23 \mathrm{~mm}$. The effective generation of $\mathrm{THz}$ pulse (in partially filled with nonlinear crystal waveguide) with high intensity makes it possible to detect $\mathrm{THz}$ radiation by Schottky diode located at the end of sufficiently long waveguide, that is $60 \mathrm{~cm}$ long.

Oscillograms of picosecond laser pulses (a) and $\mathrm{GHz}-\mathrm{THz}$ (b) pulse train at timebase $10 \mathrm{~ns} / \mathrm{mm}$ is shown in Fig.3 when the crystal is centrally-symmetrically located in the waveguide with cross-section $7.2 \times 3.6 \mathrm{~mm}^{2}$

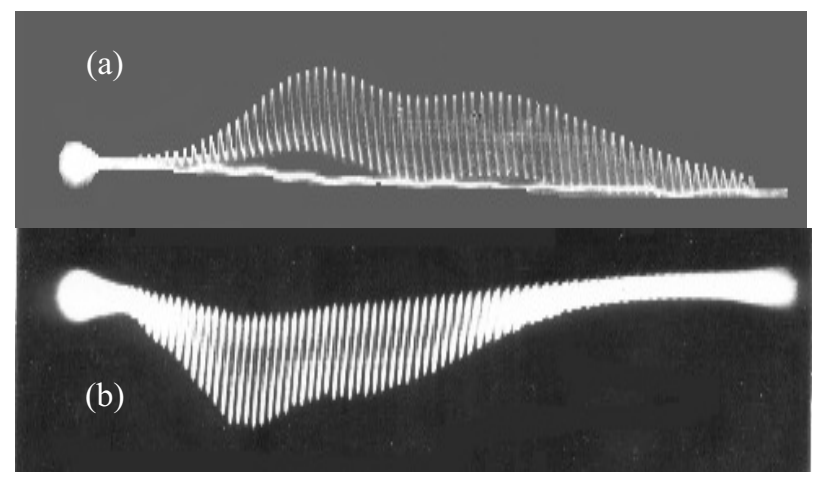

Fig.3. Oscillograms of the trains of laser (a) and $\mathrm{GHz}-\mathrm{THz}$ (b) pulses when the crystal is located centrally-symmetrically in the waveguide with cross-section $7.2 \times 3.6 \mathrm{~mm}^{2}$. 
The duration of trains is $500 \mathrm{~ns}$. The maximum peak power is $2.6 \mathrm{~W}$. Whisker-contacted Schottky diode is the preferred nonlinear element, but it works on linear part of its voltampere characteristic, because amplitude envelopes of the picosecond laser and $\mathrm{THz}$ pulse trains are similar (Fig. 3a, 3b).

$\mathrm{THz}$ picosecond pulse train with repetition pulses of $\sim 7 \mathrm{~ns}$, at timebase $5 \mathrm{~ns} / \mathrm{mm}$, and peak power $142 \mathrm{~mW}$ detected by Schottky diode in waveguide $2.4 \times 1.2 \mathrm{~mm}^{2}$ is shown in Fig.4(b).

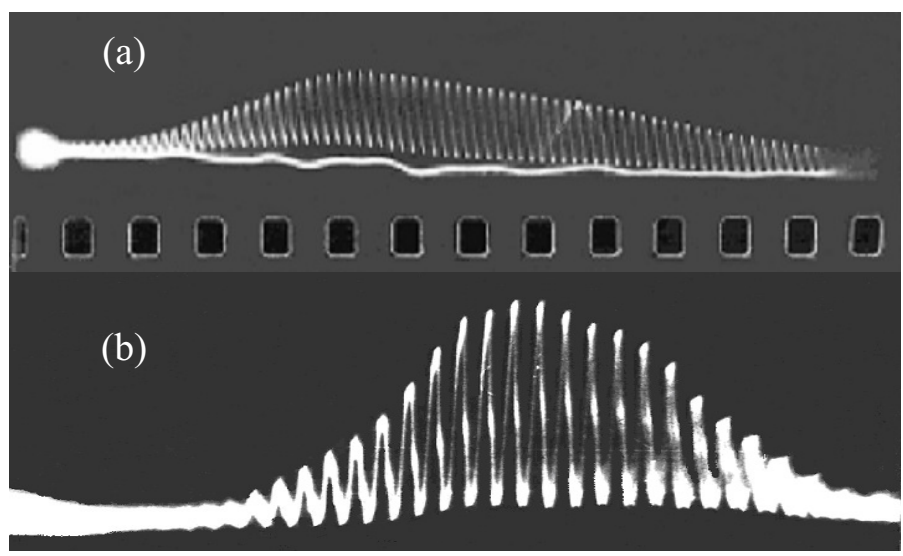

Fig.4. Oscillograms of the trains of laser (a) and THz (b) pulses when the crystal is located centrallysymmetrically in the waveguide with cross-section $2.4 \times 1.2 \mathrm{~mm}^{2}$.

\section{Conclusion}

Increasing conversion efficiency in the order of magnitude has been obtained in waveguide partially filled with $\mathrm{LiNbO}_{3}$ compared to the crystal placed in a free space ${ }^{17}$. At phase matching in waveguide efficiency energy of nonlinear conversion compared to mismatching is increased $\sim 50$ times. The quantum efficiency of energy conversion up to $28 \%$ of maximum value is obtained with Schottky diode.

For high efficiency $\mathrm{THz}$ radiation generation it is suggested ${ }^{18}$ to coherently combine $\mathrm{THz}$ radiation from nonlinear crystal-waveguide arrays. These dielectric antenna arrays can be used for implement efficient, robust, and compact $\mathrm{THz}$ sources suitable for applications such as security screening, medical imaging, quality control, chemical composition detection and package and container inspection. 


\section{References}

1. Yu.O. Avetisyan, R.M. Martirosyan, É.G. Mirzabekyan and P.S. Pogosyan, Sov. J. Quantum Electron. 8, 382 (1978).

2. A.S. Nikoghosyan, Sov. J. Quantum Electronics, (Russian) 15, N5, 969 (1988).

3. E. M. Laziev and A.S. Nikoghosyan, in SPIE, Mode-Locked Lasers and Ultrafast Phenomena, 1842, 113-118, (1991).

4. K. Wang and D.M. Mittleman, Nature, 432, 376-379 (2004).

5. A. Bandyopadhyay, A. Sengupta, V. Johnson, J. A. Harrington, J. F. Federici, in Terahertz and Gigahertz Electronics and Photonics V, Proc. SPIE 6120, 61200B, (2006).

6. Z. Ruan, G. Veronis, K. L. Vodopyanov, M. M. Fejer, S. Fan, Opt. Express. 2009 Aug 3;17 (16):13502-15 (2009).

7. S. S. Harsha, N. Laman, D. Grischkowsky, Applied Physics Letters, 94, Issue 9, id. 091118 (3 pages), (2009).

8. R. Mendis, A. Nag, F. Chen, D. M. Mittleman, Appl. Physics Letters, 97, Issue 13, id. 131106 (3 pages) (2010).

9. J. R. Knab, A J. L. Adam, R. Chakkittakandy, P. C. M. Planken, Appl. Physics Letters, 97, Issue 3, id. 031115 (3 pages) (2010).

10. A. Rusina, M. Durach, K. A. Nelson and M. I. Stockman, arXiv:0808.1324v1 [physics.optics] 9 Aug (2008).

11. C. Yeh, F. Shimabukuro, P. H. Siegel, Applied Optics IP, 44, Issue 28, 10, 5937-5946 (2005).

12. S. Bozhevolnyi and Kh. Nerkararyan, Opt.Lett. 34, 2039 (2009).

13. A. S Nikoghosyan, P. M. Martirosyan, A. A. Hakhoumian, J. M. Chamberlain, R.A. Dudley, N.N. Zinov'ev, Inter. Journal Electromagnetic Waves and Electronic System. 11, N4, 47-55 (2006).

14. N. N Zinov'ev, A. S. Nikoghosyan, and J. M. Chamberlain, in Proceedings of SPIE, 6257, 62570P1-62570P8 (2006).

15. P. A. D. Wood, W. C. B. Peatman, D. W. Porterfield and T. W. Crowe, in Proc. Fourth International Symposium on Space Terahertz Technology, (1993), p.377-391.

16. H. P. Roser, H-W. Hubers, E. Brundermann and M. F Kimmitt, Semicond. Sci. Technol. 11, 1328-1332 (1996).

17. N.N. Zinov'ev, A.S. Nikoghosyan, R.A. Dudley, J.M. Chamberlain. Physical Review B, 76, 235114-1235114-16 (2007).

18. D. Creeden, P. A. Ketteridge, Y. E. Young, R. Thompson, USA Patent No.: 7787724B2 (2010). 